\title{
Mode-selective dynamic light scattering: theory versus experimental realization
}

\author{
Thomas Gisler, Heinrich Rüger, Stefan U. Egelhaaf, Jürg Tschumi, \\ Peter Schurtenberger, and Jaroslav Rička
}

\begin{abstract}
We present a quantitative experimental comparison of fiber-based, single- and few-mode dynamic light scattering with the classical pinhole-detection optics. The recently presented theory of mode-selective dynamic light scattering [Appl. Opt. 32, 2860 (1993)] predicts a collection efficiency and a signal-tobaseline ratio superior to that of a classical pinhole setup. These predictions are confirmed by our experiments. Using single-mode optical fibers with different cutoff wavelengths and commercially available mechanical components, we have constructed a mode-selective detection optics in a simple and compact dynamic light-scattering spectrometer that permits an optimal compromise between signal intensity and dynamical resolution.
\end{abstract}

\section{Introduction}

The compactness and small size of fiber-based optical devices have always attracted designers of dynamic light-scattering (DLS) experiments (see, e.g., Refs. 1-3). In early DLS applications, multimode fibers were used merely as a convenient and flexible means of light transportation, whereas the spatial filtering needed to meet the coherence requirements was conventionally accomplished by the use of pinholes. However, a new development was initiated by Brown, ${ }^{4}$ who for the first time discussed the application of single-mode fibers to DLS.

A single-mode fiber ${ }^{5}$ is an optical waveguide whose very small core radius (typically $2 \mu \mathrm{m}$ ) is comparable with the wavelength of light. More precisely, a waveguide becomes single mode if the operating wavelength, $\lambda$, exceeds a certain characteristic cutoff wavelength, $\lambda_{c}$. Above $\lambda_{c}$ only one transverse mode of the electric field (disregarding the polarization) is

T. Gisler is with the Institut für Terrestrische Oekologie, Federal Institute of Technology (ETH), Grabenstrasse 3, CH-8952 Schlieren, Switzerland. H. Ruger and P. Schurtenberger are with the Institut für Polymere, and S. U. Egel haaf is with the Laboratorium für Elektronenmikroskopie I, Federal Institute of Technology (ETH), ETH-Zentrum, CH-8092 Zürich, Switzerland. J . Tschumi and J . Rička are with the Institut für Angewandte Physik, University of Bern, Sidlerstrasse 5, CH-3012 Bern, Switzerland.

Received 6 September 1994; revised manuscript received 13 December 1994.

0003-6935/95/183546-08\$06.00/0.

(1) 1995 Optical Society of America. propagated, i.e., thetransversestructure of the guided field is determined by the characteristics of the waveguide. As the propagated mode is perfectly transversely coherent, spatial pinhole filtering as required in the use of multimode DLS receivers is superfluous with single-mode fibers. This permits the construction of receivers of ultimate simplicity; in principle, a bare single-mode fiber without any additional optical components is sufficient. ${ }^{6}$ At the same time, welldesigned single-mode receivers provide a lightcollection efficiency superior to that of conventional pinhole optics. However, the superb performance of single-mode fiber receivers is still to be recognized by a wider audience, because early comparisons of fiber optical and classical pinhole receivers, ${ }^{1,4,7}$ together with a lack of a quantitative theoretical understanding, have produced controversial results about the general applicability of single-modefiber optical recei vers in DLS.

Recently, the theoretical basis of DLS with singlemode as well as with few-mode receivers was elaborated, ${ }^{3}$ and this now permits a rigorous quantitative comparison of different types of setups. The performance of a DLS setup can be expressed in terms of two quantities. The first quantity is the lightcollection efficiency, $\langle J\rangle / \mathrm{J}$ e, where $\langle J\rangle$ is the average power obtained from a given sample and $J \mathrm{e}$ is the power of the excitation beam. The second quantity is the coherence factor, f, i.e., the prefactor of the dynamic part of the normalized signal correlation function, $g^{2}(\tau)$, that obeys the generalized Siegert relation, $g^{2}(\tau)=1+f\left|g^{1}(\tau)\right|^{2}$. 
This paper is organized as follows. In Section 2 we review the relevant expressions for the light-collection efficiency and the coherence factor for threetypes of DLS receivers, i.e., the single- and few-mode receivers and the classical pinhole setup. For a detailed derivation of the basic formulas we refer to Ref. 3 . The explicit expressions of the general multimode case tend to be lengthy; we therefore provide predictions for a simplified model experiment. The experimental section, Section 3, is divided into two parts. First, we compare the theoretical predictions with data from a model setup suitable for an unambiguous quantitative comparison of different types of receivers. Second, we present an example of a practical realization of a single and few-mode setup and discuss its performance.

\section{Theory}

\section{A. Single-Mode Fiber Receiver}

The key feature of a single-mode fiber receiver is its selection of a single scattered field component that matches the so-called receiver mode. ${ }^{3}$ This auxiliary el ectromagnetic fiel d can be computed as the continuation of the fiber mode out of the fiber front face. It completely describes the spatial receiving characteristics of a single-mode receiver. At first glance the receiver mode might appear as a purely mathematical construction; nevertheless, it is easily made visible: one only has to couple a laser beam into the detector end of the fiber and observe the light beam that emerges from the receiving end of the setup. This beam is indicated in Fig. 1(a). In a typical DLS setup the receiver beam is collimated or moderately focused into the center of the sample cell by means of a suitablelens in front of the fiber. Because the fundamental fiber mode is well approximated by a Gaussian beam, the receiver beam too should have a Gaussian profile. (Poor optical quality or an insufficiently large aperture of the focusing lens results in diffraction rings and subsequent coupling losses.) The amplitude profile of the receiver beam is given by

$$
\mathrm{B}(\mathbf{r})=\mathrm{B}(\mathrm{x}, \mathrm{y})=\exp \left[-\left(\mathrm{x}^{2}+\mathrm{y}^{2}\right) / 2 \mathrm{a}_{0}{ }^{2}\right],
$$

where $a_{0}$ is the characteristic beam radius and $(x, y)$ are coordinates perpendicular to the beam axis [see Fig. 1(b) for an outline of the scattering geometry]. The corresponding intensity profile is thus given by $|B(\mathbf{r})|^{2}=\exp \left[-\left(x^{2}+y^{2}\right) / a_{0}{ }^{2}\right]$. (Note that another frequently encountered characteristic measure of the size of a Gaussian beam is the so-called waist radius, $\mathrm{w}_{0}=\sqrt{2} \mathrm{a}_{0}$.) In a well-al igned setup the optical axis of the receiver beam intersects the optical axis of the probing laser beam, and both axes span the scattering plane I. Usually the probing laser beam can al so be approximated by a Gaussian beam. We denote its amplitude profile by

$$
A(\mathbf{r})=A\left(x, y^{\prime}\right)=\exp \left[-\left(x^{2}+y^{\prime 2}\right) / 2 a_{e}^{2}\right],
$$

where $a_{e}$ is the effective radius of the excitation beam

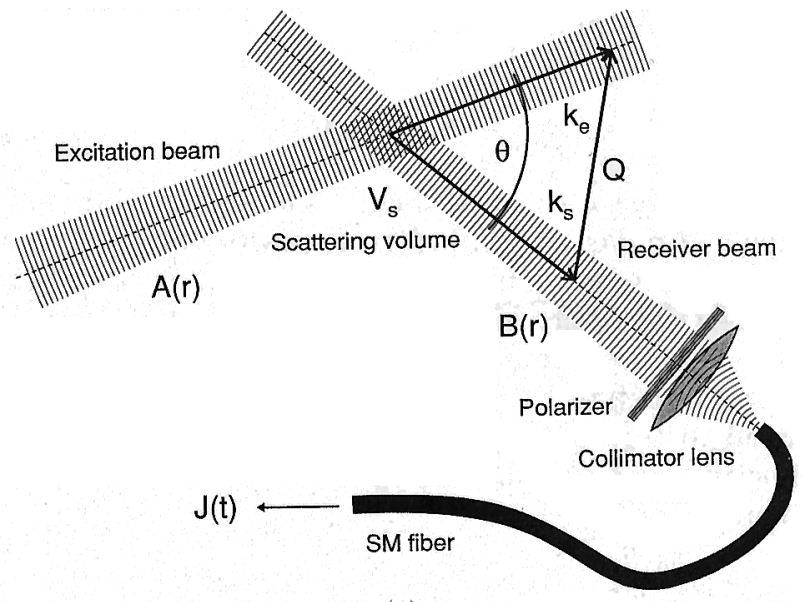

(a)

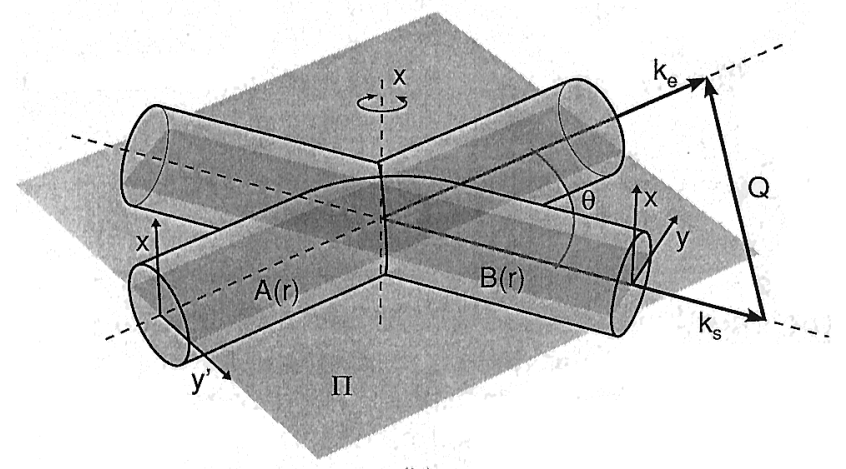

(b)

Fig. 1. (a) Schematic representation of a light-scattering experiment with a single-mode $(\mathrm{SM})$ receiver. The scattering volume is illuminated by excitation beam $A(\mathbf{r})$, which is intersected by observation beam $B(\mathbf{r})$. The collimator lens in front of the fiber is used to modify the receiver beam characteristics such that a reasonably small effective beam radius and a suitable working distance between the receiver and the scattering volume can be realized. The polarizer may be left out for optically isotropic scattering samples. (b) Scattering geometry and coordinatesystem. Beam profiles $A(\mathbf{r})$ and $B(\mathbf{r})$ of the excitation beam and of the observation beam, respectively, are shown as cylinders whose intersection forms scattering volume V. This situation corresponds to a well-aligned setup with moderately focused beams. The polarization of the excitation beam is perpendicular to scattering plane $\Pi$ spanned by the wave vectors $\mathbf{k}_{\mathrm{e}}$ and $\mathbf{k}_{\mathrm{s}}$. Angle $\theta$ between the excitation beam and the observation beam determines scattering vector $\mathbf{Q}=\mathbf{k}_{\mathrm{s}}-\mathbf{k}_{\mathrm{e}}$. Coordinates $(\mathrm{x}, \mathrm{y})$ and $\left(\mathrm{x}, \mathrm{y}^{\prime}\right)$ are perpendicular to the axis of beam propagation.

and $\left(\mathbf{x}, \mathbf{y}^{\prime}\right)$ denote coordinates perpendicular to the direction of propagation of the excitation beam.

Product $\mathrm{A}(\mathbf{r}) \mathrm{B}(\mathbf{r})$ determines the size and shape of the scattering volume. For the sake of simplicity we assume that the probing laser beam is polarized in the $x$ direction perpendicular to the scattering plane; in addition, the observed sample is assumed to be optically isotropic so that the experimentalist is dispensed from using a polarizer in front of the receiving fiber.

1. Light-Collection Efficiency

Characterizing the sample by its Rayleigh ratio, $R(\theta)$, one can write the average predetection signal (optical 
power impinging on the detector) obtained from a loss-free single-mode receiver as

$$
\left\langle J=R(\theta) I_{\mathrm{e}} \Omega_{0} \mathrm{~V}_{0}(\theta) .\right.
$$

This expression exactly corresponds to the wellknown standard formula for the power obtained from a scattering system such as it would follow from geometrical optics. However, factors $\mathrm{I}_{\mathrm{e}}, \Omega_{0}$, and $\mathrm{V}_{0}$ are to be interpreted in the frame of wave optics of Gaussian beams. The effectiveintensity, $I_{e}$, of excitation in terms of the excitation power, $\mathrm{J} e$ and the effective cross section, $\pi a_{e}{ }^{2}$, of the excitation beam are given by

$$
\mathrm{I}_{\mathrm{e}}=\frac{\mathrm{J} \mathrm{e}}{\pi \mathrm{a}_{\mathrm{e}}^{2}} \quad \text { where } \pi \mathrm{a}_{\mathrm{e}}^{2}=\int\left|\mathrm{A}\left(\mathrm{x}, \mathrm{y}^{\prime}\right)\right|^{2} \mathrm{dxdy^{ \prime }} .
$$

The effective solid angle of observation, $\Omega_{0}$, results as

$$
\Omega_{0}=\frac{\lambda^{2}}{\pi \mathrm{a}_{0}^{2}} \quad \text { where } \pi \mathrm{a}_{0}^{2}=\int|\mathrm{B}(\mathrm{x}, \mathrm{y})|^{2} \mathrm{dxdy} .
$$

Here $\lambda$ is the wavelength (in the sample) and $\pi a_{0}{ }^{2}$ is the effective cross section of the observation beam. Note that $\Omega_{0}$ is not determined by the aperture of the collimator lens but rather by the divergence of the Gaussian observation beam that is focused to radius $a_{0}$.

Finally, the scattering volume, $V_{0}$, is defined as

$$
\mathrm{V}_{0}=\int|\mathrm{B}(\mathbf{r}) \mathrm{A}(\mathbf{r})|^{2} \mathrm{~d}^{3} \mathrm{r}
$$

In this case of a well-aligned Gaussian beam one obtains

$$
V_{0}(\theta)=\frac{\pi^{3 / 2}}{\sin \theta}\left(\frac{a_{e}{ }^{4} a_{0}{ }^{4}}{a_{e}{ }^{2}+a_{0}^{2}}\right)^{1 / 2}
$$

On inserting the results from Eqs. (4), (5), and (7) into Eq. (3), one obtains the signal from a well-aligned loss-free single-mode receiver as

$$
\left\langle J=J e^{R}(\theta) \frac{\lambda}{\sqrt{\pi} \sin \theta}\left(\frac{\lambda^{2}}{a_{e}^{2}+a_{0}^{2}}\right)^{1 / 2} .\right.
$$

From Eq. (8) we deduce an important rule: while keeping excitation power $\mathrm{J}$ e constant, one can increase the received signal by focusing both the observation beam and the excitation beam to a smaller spot. There are, however, fundamental and practical limits. First, the Gaussian-beam approximation employed in the derivation of Eq. (8) is applicable only if both $a_{e}$ and $a_{0}$ are much larger than the illuminating wavelength. Second, in practical setups one is usually limited to a working distance of $\sim 10 \mathrm{~cm}$, and this limits $a_{e}$ and $a_{0}$ to a few tens of micrometers. Third, it is sometimes desirable to sacrifice sensitivity for stability. A setup is much less sensitive to misalignment or disturbances if the observation beam is collimated to a diameter of $\sim 1 \mathrm{~mm}$ instead of being focused. To assess the effect of misalignment, we note here that scattering volume $V_{0}(d)$ resulting from a vertical displacement $d$ between the excitation beam and the observation beam varies as $V_{0}(d)=$ $V_{0} \exp \left[-d^{2} /\left(a_{e}^{2}+a_{0}^{2}\right)\right]$, where $V_{0}$ is given by $E q .(7)$.

\section{Coherence Factor}

The field component selected by a single-mode receiver is perfectly transversely coherent, and this implies a maximal coherence factor $f=1$, independent of the size and shape of scattering volume and scattering angle. A slight decrease below this theoretical value may be caused by any of the following effects.

1. The receiver picks up some static stray light, most likely at small and large scattering angles.

2. There is a substantial incoherent background caused by solvent scattering.

3. The signal is too high, and the resulting detector dead time causes saturation effects. ${ }^{8}$ (The signal at large and small scattering angles may be very high because of the large scattering volume.)

4. The operating wavel ength, $\lambda$, is too close to the cutoff wavelength, $\lambda_{c}$, of the fiber, and higher-order modes al ready begin to appear.

5. The fiber is too short and transmits spurious cladding modes. If a truly single-mode operation is desired, one should use at least $3 \mathrm{~m}$ of fiber.

6. The coherence length of the laser is comparable with the size of scattering volume in the direction of the scattering vector, $\mathbf{Q}$. With certain diode lasers this effect may occur at large scattering angles.

\section{B. Few-Mode Fibers}

When the fiber core is enlarged such that $\lambda_{c}$ exceeds operating wavelength $\lambda$, the fiber starts to propagate fields that exhibit a variety of amplitude profiles. However, all of these profiles can be expressed as linear combinations of a certain finite set of eigenmodes. A convenient choice of eigenmodes are the so-called LP ${ }_{I m}$ modes. These linearly polarized (LP) modes are extensively discussed in Ref. 5. At a constant operating wavel ength, the number of propagated LP modes increases with increasing core radius (i.e., with $\lambda_{c}$; seeF ig. 2). Here we restrict ourselves to few-mode fibers that propagate, practically without losses, only a small number of modes. In a few-mode fiber receiver, each of the guided modes corresponds to one partial observation beam. Each of these beams exhibits one characteristic amplitude profile, $B_{i}(\mathbf{r})$; the subscript $i=0$ denotes the fundamental Gaussian mode. Observation beams resulting from the LP modes are well approximated by the so-called Laguerre-Gaussian beams. ${ }^{5}$

An essential feature of the LP modes is their 


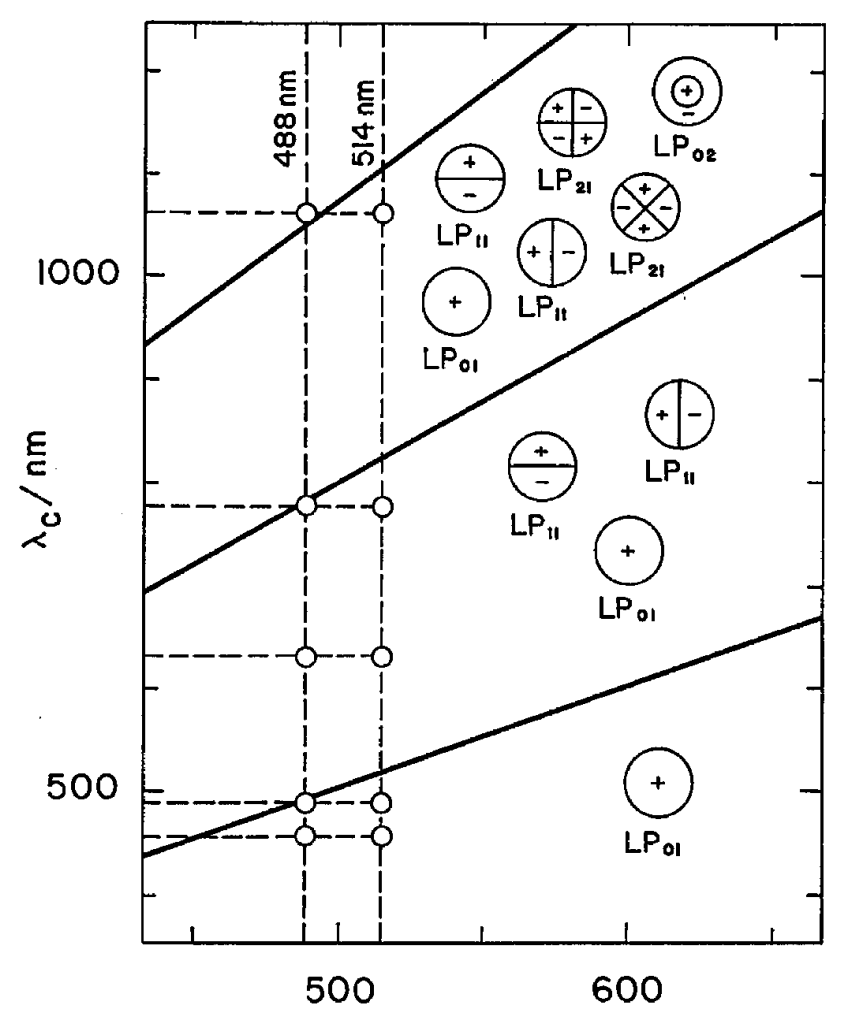

$\lambda / \mathrm{nm}$

Fig. 2. Chart to help determine the number of eigenmodes that can be propagated through a given fiber at a given operating wavelength, calculated by the use of theoretical expressions for step-index fibers. ${ }^{5}$ The vertical axis indicates single-mode cutoff wavelength $\lambda_{c}$ of the given fiber. For example, a fiber with $\lambda_{c}=$ $488 \mathrm{~nm}$ propagates only the fundamental LP $\mathrm{P}_{01}$ mode when the operating wavelength exceeds $488 \mathrm{~nm}$. The dashed horizontal lines represent $\lambda_{c}$ of five commercially available fibers, and the two dashed vertical lines correspond to two common laser lines. The solid curves separate regions with one, three, and six propagating modes. For example, when a fiber with $\lambda_{c}=633 \mathrm{~nm}$ is operated at $514 \mathrm{~nm}$, it will propagate three LP modes. Note that with real fibers the borders between the different regions are not as perfectly sharp as suggested by the chart. The open circles schematically indicate the amplitude profiles of the L $P_{I m}$ modes and illustrate the meaning of the indices I and $\mathrm{m}$ : the first index is the azimuthal mode number, i.e., the number of maxima encountered when circling around the fiber axis, and the second index indicates the number of maxima counted in the radial direction.

orthogonality. This property implies that

$$
\int B_{i}(x, y) B_{j}^{*}(x, y) d x d y=\pi a_{i}^{2} \delta_{i j}
$$

where $\pi a_{i}^{2}=\int\left|B_{i}(x, y)\right|^{2} d x d y$ is the effective cross section of beam $\mathrm{i}$ and $\delta_{\mathrm{ij}}$ is the Kronecker symbol. Consequently, the total power transmitted through a few-mode fiber is simply the sum of contributions of the individual modes (there areno interferenceterms):

$$
J=\sum_{i=0}^{N-1} J i
$$

Here $\mathrm{N}$ is the number of guided modes and $\mathrm{J}_{\mathrm{i}}$ is the partial power carried by the ith fiber mode.

\section{Light-Collection Efficiency}

The partial average power obtained from one of the observation beams is given by Eq. (3). To calculate the partial quantities $\Omega_{\mathrm{i}}$ and $\mathrm{V}_{\mathrm{i}}$, one inserts profile $\mathrm{B}_{i}(\mathbf{r})$ into Eq. (5). Together with Eq. (10), the average signal of a few-mode receiver is given by

$$
\langle\rangle=\sum_{i=0}^{N-1}\langle i\rangle=R(\theta) I_{e} \sum_{i=0}^{N-1} \Omega_{i} V_{i}
$$

We remind the reader that for this result the assumption of uniform transmission efficiencies for all modes has been made; this assumption is equivalent to the ideal loss-free fiber. It is convenient to re-express Eq. (11) in terms of power $J \mathrm{~J}$ ) from the fundamental mode and of the effectivenumber of modes, $\mathcal{N}$ :

$$
\langle J\rangle=\left\langle J_{0}\right\rangle \mathcal{N} \quad \text { where } \mathcal{N}=\frac{1}{\Omega_{0} \mathrm{~V}_{0}} \sum_{\mathrm{i}=0}^{N-1} \Omega_{\mathrm{i}} \mathrm{V}_{\mathrm{i}}
$$

Note that in general one has to expect an effective number of modes that is smaller than the number of the guided modes:

$$
\mathcal{N} \leq \mathrm{N} .
$$

This is because the fundamental Gaussian beam can be focused best; it is a minimum uncertainty beam. (An additional reason for $\mathcal{N}<\mathrm{N}$ may be that an insufficient aperture of the focusing lens causes losses for higher-order modes.) For the Laguerre-Gaussian beams, quantities $\Omega_{\mathrm{i}} \mathrm{V}_{\mathrm{i}}$ may be explicitly calculated from E qs. (5) and (6). The resulting expressions for higher-order beams are quite lengthy, but matters are substantially simplified if the vertical size of the excitation beam is much larger than $\mathrm{a}_{0}$. We call this case the limit of a laterally homogeneous source. ${ }^{3}$ In this limit partial average powers $\left({ }_{i}\right)$ are independent of observation profiles $B_{i}(\mathbf{r})$, and the number of effective modes equals the number of guided modes, i.e.,

$$
\mathcal{N}=\mathrm{N} .
$$

Each of the modes carries the same average power, and the total average signal is given by

$$
\left\langle J=J_{0} \mathcal{N} \quad \text { where } J_{0}=J_{e} R(\theta) \frac{\lambda}{\sqrt{\pi} \sin \theta} \frac{\lambda}{a_{e}} .\right.
$$

The limit of a laterally homogeneous source does not represent the optimum for a practical setup, but it is useful for the comparison of different types of receivers.

\section{Coherence F actor}

The autocorrelation function of signal $\mathrm{J}(\mathrm{t})$ can be expressed in terms of partial autocorrelation and 
cross-correlation functions of mode contributions $\mathrm{J}_{\mathrm{i}}(\mathrm{t})$ :

$$
\left.G(\tau)=\sum_{i, j=0}^{N-1} G_{i j}(\tau)=\sum_{i, j=0}^{N-1} J_{i}(0) J_{j}(\tau)\right\rangle .
$$

This leads to the following expression for coherence factor f:

$$
f=\frac{1}{\mathcal{N}^{2}} \sum_{i, j=0}^{N-1}\left|W_{i j}\right|^{2},
$$

where $\mathrm{W}_{\mathrm{ij}}$ are normalized cross-overlap coefficients defined as

$$
W_{i j}=\frac{a_{0}^{2} V_{i j}}{a_{i} a_{j} V_{0}} \text { where } V_{i j}=\int|A(r)|{ }^{2} B_{i}(\mathbf{r}) B_{j}^{*}(\mathbf{r}) d^{3} r \text {. }
$$

In the limit of a laterally homogeneous source the cross terms in the numerator of Eq. (17) vanish by virtue of the orthogonality of the modes, and one obtains

$$
f=\frac{1}{N} .
$$

It should be noted that this simple relation between the number of guided modes, $\mathrm{N}$, and the coherence factor, f, applies strictly for a laterally homogeneous source only. Nevertheless, there are strong indications that, in general,

$$
\frac{1}{N} \leq \mathrm{f} \leq \frac{1}{\mathcal{N}} .
$$

However, this relation has yet to be proved.

\section{Optimization of a Few-ModeReceiver}

The light-collection efficiency increases with the effective number of modes $\mathcal{N}$, whereas coherence factor $\mathrm{f}$ decreases with $\mathcal{N}$. In the optimal case, which we call a mode-selective receiver, one obtains $f=1 / \mathcal{N}$. The signal-to-noise ratio of a DLS experiment increases with $\mathcal{N}$ but decreases with decreasing f. Thus one may ask for a value of $\mathcal{N}$ that optimizes both $f$ and the signal-to-noise ratio. For a mode-selective receiver, the signal statistics are characterized by a gamma distribution. According to the rigorous analysis by Schätzel, ${ }^{9}$ the signal-to-noi se-ratio can be improved at small lag times $\tau$ by a suitable choice of $N$, although only up to a factor of $\sqrt{2}$. In addition, an improvement only can be expected for an ideal sample and with an ideal laser: the advantage of a few-mode receiver vanishes and even becomes a disadvantage if the incoherent background (scattering from the solvent) is large or if the laser is affected by instabilities in intensity, wavelength, or beam-point position. In such nonideal situations the use of a single-mode fiber represents the optimal choice. A few-mode receiver, however, is useful if the rapid estimation of the average signal is also of interest, i.e., if the receiver should be optimized for both dynamic and static light-scattering experiments.

\section{Classical Pinhole Setup}

A classical pinhole receiver that consists of a lens imaging the excitation beam onto a detector aperture can be considered as a multimode receiver that accepts a large number $\mathrm{N}$ of modes. However, the accepted modes are subject to great coupling losses, and thus the effective number of modes $\mathcal{N}$ is small. ${ }^{3}$ Here we present the pinhole receiver only for the purpose of comparison with the fiber receivers. This comparison can be done best for a laterally homogeneous source. In this limit the average signal from the pinhole receiver can be written as

$$
\langle\mathrm{J}\rangle=\mathrm{J}_{0} \mathcal{N},
$$

where $\mathrm{J}_{0}$ is the same quantity as in Eq. (15), whereas the effective number of modes $\mathcal{N}$ is given by the well-known expression

$$
\mathcal{N}=\frac{A_{D}}{A_{\text {coh }}} .
$$

Here $A_{D}$ is the area of detector aperture $P_{L}$, and $A_{\text {coh }}=$ $\left.\lambda^{2}\right|^{2} / \pi a_{L}{ }^{2}$ is known as the area of coherence; quantity $a_{L}$ is the radius of the lens aperture and I denotes the distance between the lens and the scattering volume.

In Ref. 3 it was shown that a good approximation to the corresponding coherence factor is

$$
f=1 /(\mathcal{N}+1) \text {. }
$$

It should be noted that both Eqs. (22) and (23) apply strictly for a laterally homogeneous source only. In the present context this limit implies that the detector pinhole is, in a statistical sense, homogeneously illuminated. The general case of an excitation beam of finite size is considerably more complicated.

\section{Fibers Versus Pinholes}

Comparing Eqs. (15), (19), (21), and (23), we arrive at the following important result: the coherence factor of a few-mode receiver is always larger than the coherence factor of a classical pinhole receiver working with the same effective number of modes, i.e., with the same power. A strikingillustration of the advantage of the fiber receiver is the following numerical example: a single-modereceiver with a perfect coherence factor of $f=1$ provides 10 times more power than a pinhole receiver working with $\mathrm{f}=0.9$.

\section{Experimental}

To test theoretical expressions (19), (23), (15), and (21) for the coherence factor and the average signal, we performed DLS experiments by using a classical pinhole and a fiber-optic receiver unit. The experimental details are presented in Subsection 3.A. In 


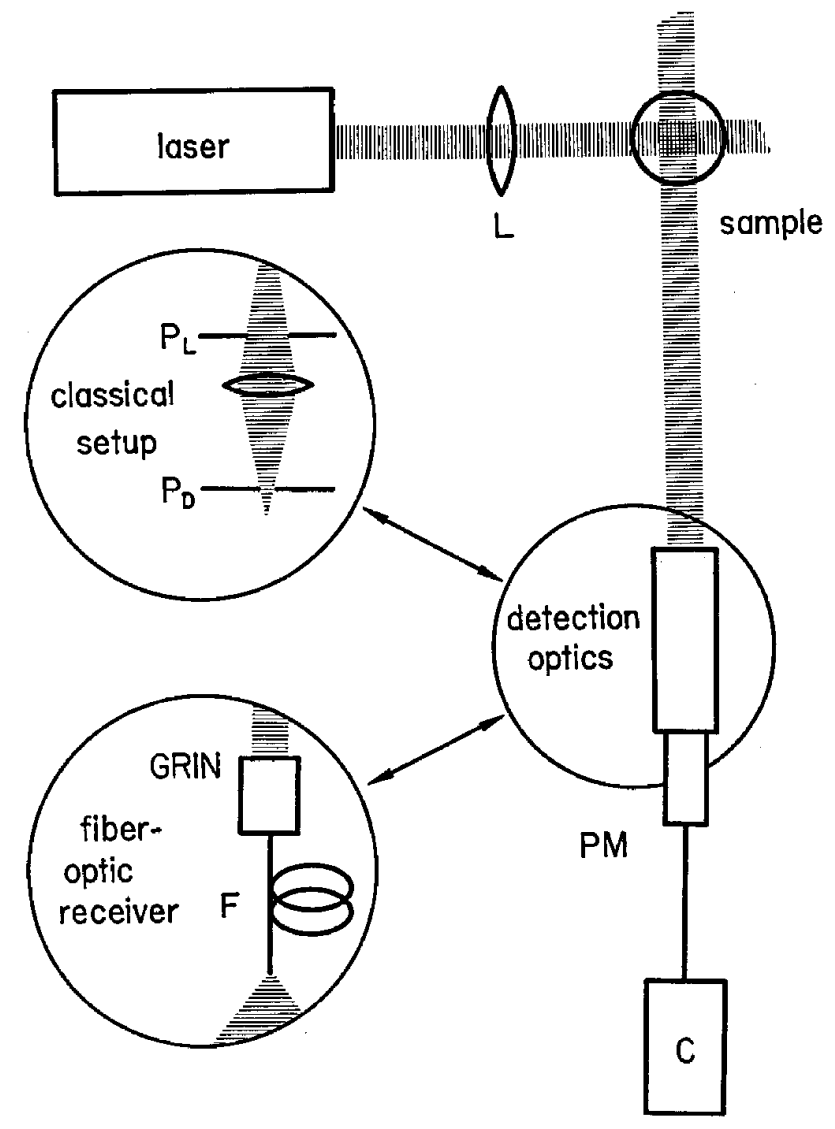

Fig. 3. DLS setup used to compare the classical pinhole and a fiber-optic receiver. The laser beam is expanded vertically by cylindrical lens $L$ to approximate the limit of a laterally homogeneous source. In the classical pinhole receiver the scattering volume is imaged by the combination of the apertures $P_{L}$ and $P_{D}$ and the lens onto the photomultiplier tube (PM). In the fiber-optic receiver the scattering volume and the direction of the received mode is defined by the very small numerical aperture of the GRIN lens, which is used to couple the scattered light into fiber F. In both cases the photomultiplier signal is fed into correlator $\mathrm{C}$.

Subsection 3.B we present a fiber-optic spectrometer that uses fibers with different cutoff wavelengths to vary the number of guided modes, permitting an optimization of the coherence factor and the average signal for an individual experimental situation.

\section{A. Comparison Pinhole: Fiber Setup}

The comparison of the pinhole setup with the fiberoptic setup was carried out with the setup schematically shown in Fig. 3. To provide a uniform laser signal over a large area, i.e., to realize the case of a laterally homogeneous source, we vertically expanded the excitation beam with a cylindrical lens to $\sim 5 \mathrm{~mm}$. The vertically polarized $\mathrm{Ar}^{+}$laser (Lexel) was operated at $514.5 \mathrm{~nm}$. Scattered light from a dilute suspension of polystyrene latex spheres with diameters of $87 \mathrm{~nm}$ was collected at a scattering angle of $\theta=90^{\circ}$ by a pinhole or by fiber receivers.
Table 1. Parameters of the Fiber Assemblies

\begin{tabular}{crccc}
\hline Manufacturer & $\begin{array}{c}\lambda_{c} \\
(\mathrm{~nm})\end{array}$ & $\mathrm{N}^{\mathrm{a}}$ & $\begin{array}{c}\text { Focal Length } \\
\text { of Collimator Lens } \\
(\mathrm{mm})\end{array}$ & $\begin{array}{c}\text { Effective } \\
\text { Radius } \mathrm{a}_{0} \\
(\mathrm{~mm})\end{array}$ \\
\hline York & 458 & 1 & 4.0 & 0.30 \\
OZ & 488 & 1 & 1.6 & 0.13 \\
OZ & 633 & 3 & 1.8 & 0.14 \\
OZ & 780 & 3 & 2.0 & 0.15 \\
OZ & 1060 & 6 & 2.5 & 0.19 \\
\hline
\end{tabular}

aThe number of guided modes is given for the operating wavelength of $514.5 \mathrm{~nm}$, based on the chart in Fig. 2.

For the fiber setup we used commercially available fiber assemblies consisting of collimating entrance lenses, which were conventional microlenses or graded index (GRIN) lenses, and fibers with cutoff wavelengths of 458 (York VSOP Ltd.), 488, 633, 780, and $1060 \mathrm{~nm}$ (OZ Optics). The parameters of these assemblies, in particular their numbers of guided modes, are shown in Table 1. In the pinhole setup the number of coherence areas was varied with the aperture of the pinhole.

The signal was detected by the use of a photomultiplier tube (Thorn EMI 9863 B/100). As the sensitivity of the photomultiplier cathode varies with position, we took precautions to ensure that the illumination profile on the photocathode was the same with all investigated receiver setups. This is crucial for the present comparative experiment. Autocorrelation function $g^{2}(\tau)$ was recorded with an ALV-5000 digital correlator. Coherence factor $f$ was calculated by a second-order cumulant fit to the experimental correlation function.

The exact value of the quantum efficiency of the detector is difficult to determine, and the measured quantity is theaverage count rate, $J \mathrm{c}$, taken from the monitor channels of the correlator, rather than the optical power, J ). This modifies Eqs. (15) and (21) to

$$
\langle\mathrm{J}\rangle=\mathrm{KJ}_{0} \mathcal{N},
$$

where constant $\mathrm{KJ} 0$ is common to all measurements. Constant $\mathrm{KJ}$ o was obtained from a least-squares fit of Eq. (23) to the experimental values of $f$ obtained with the pinhole receiver (see $\mathrm{Fig}$. 4). This value of $\mathrm{KJ} 0$ was then used to predict the coherence factor and the average signal as a function of the (discrete) number of guided modes of the mode-selective few-mode fiber setups (filled circles in Fig. 4).

Different numbers of guided modes were then realized by the use of fibers with different cutoff wavelengths (triangles in Fig. 4). The experimental data agree well with the calculated coherence factors and average signals. Small deviations are attributed to nonideality, i.e., different losses at the lenses used in the pinhole and in the fiber receiver, respectively. Figure 4 also shows that for a given average signal, the coherence factor obtained by few-mode detection is al ways higher than that obtained by pinhole detec- 


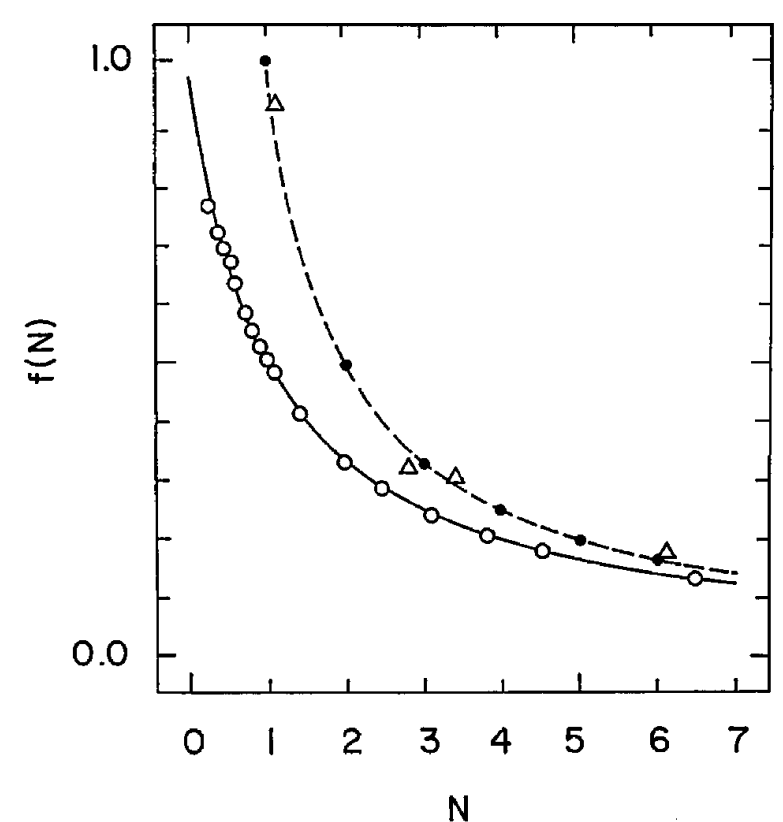

Fig. 4. Comparison of mode-selective and classical detection setup. Coherence factor $f(N)$ of the normalized intensity autocorrelation function is plotted versus the normalized average signal $\mathrm{N}$. The open circles represent the experimental data measured with the pinhole setup. The solid curve was calculated by the use of proportionality factor $\mathrm{KJ} 0$, obtained by the fit of the experimental data to Eq. (23). All experiments were performed with the same photomultiplier. The prediction for the coherence factor and the average signal for the fiber-optic detection at different cutoff wavel engths (resulting in different numbers of guided modes) is represented by the filled circles. The dashed curve serves as a guide to the eye. Triangles show experimental data measured with fibers of different cutoff wavelengths.

tion. Whereas in a classical pinhole setup one can increase the resolution of the correlation function only by permitting a drastic decrease in collection efficiency, the use of few-mode fibers al lows the experimentalist to optimize the coherence factor and the average signal over a much larger range simply by the choice of an optical fiber with an appropriate cutoff wavelength. These results are now used in the following subsection, which describes the construction of a compact fiber-optic DLS spectrometer.

\section{B. Fiber-Optic Realization}

We have constructed a simple fiber-based DLS spectrometer by using an index-matching vat and a computer-controlled rotational stage (Newport 496) as a goniometer. The excitation beam is launched into the sample by a Dantec $60 \times 30$ polarizationpreserving optical fiber. A built-in collimator in front of the launching fiber focuses the beam to a radius of $0.4 \mathrm{~mm}$ in the center of the sample cell (see Fig. 5). The excitation beam is vertically polarized and the $\mathrm{Ar}^{+}$laser (coherent) is operated at $\lambda=488$ $\mathrm{nm}$.

A small part of the excitation beam intensity is deflected by a glass plate onto a diode that al lows us to monitor the excitation beam intensity. The scat-

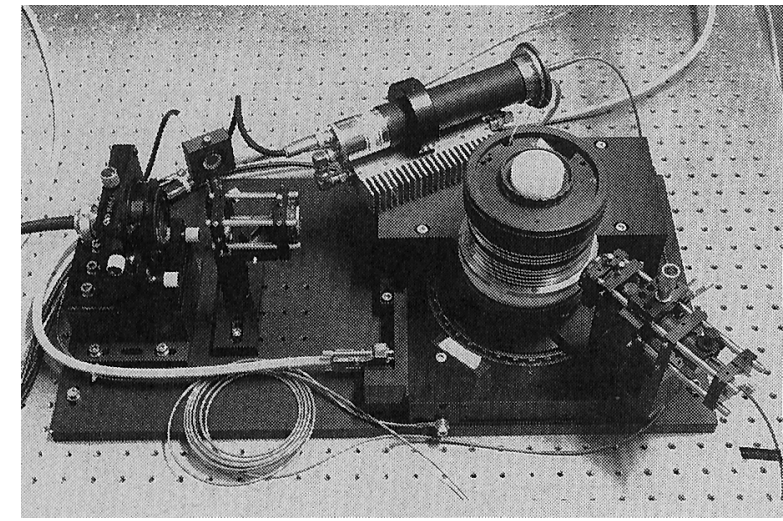

Fig. 5. Fiber-optic spectrometer. Theexcitation beam is launched by a Dantec fiber through a polarizer (left) onto the sample inserted in the cylindrical index-matching vat. The receiver optics (right) consists of a polarizer and an optical fiber mounted on the goniometer arm of the rotational stage. The entire optical components of the spectometer are mounted on an aluminum plate $57 \mathrm{~cm}$ long and $30 \mathrm{~cm}$ wide.

tered light is received by single- and few-mode fibers. They can be positioned on the goniometer by the use of a commercial optical bench (Spindler \& Hoyer Microbench). The setup is a modular design composed of commercially available components that are relatively inexpensive. The goniometer is controlled through an external routine, which can be called from within theALV software. This permits the automatization of both static and dynamic light-scattering measurements at different angles. The fiber is held by a home-built metal sleeve with variable vertical tilt angle mounted on an $x-y$ translation stage. The flexibility and the negligible transmission loss in the optical fibers permits the detection unit (in this case

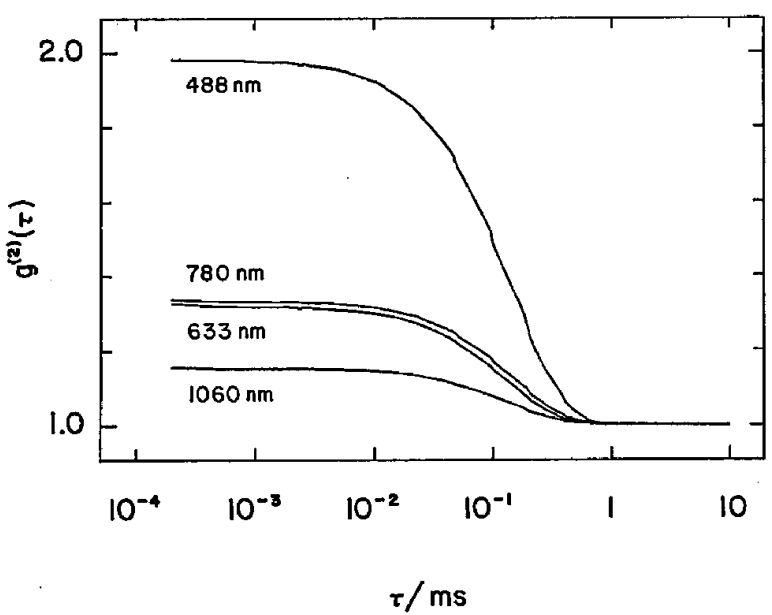

Fig. 6. Normalized intensity autocorrelation functions $g^{2}(\tau)$ at $\theta=90^{\circ}$, measured with the fiber-optic goniometer setup described in Subsection 3.B. The sample was a dilute suspension of polystyrene latex spheres of $91 \mathrm{~nm}$ diameter. Fibers with cutoff wavelengths of 488, 633, 780, and $1060 \mathrm{~nm}$ were used. Each correlation function is the average of five individual measurements of a duration of $600 \mathrm{~s}$. 
Table 2. Comparison of the Different Fibers in the Experimental Realization

\begin{tabular}{rrrrr}
\hline $\begin{array}{c}\lambda_{c} \\
(\mathrm{~nm})^{\mathrm{a}}\end{array}$ & $\mathrm{N}^{\mathrm{b}}$ & $\mathrm{N}_{\exp }{ }^{\mathrm{c}}$ & $\mathbf{f}(\mathbf{N})^{\mathrm{d}}$ & $\mathrm{f}_{\exp }$ \\
\hline 488 & 1 & 1.00 & 1.000 & 0.982 \\
633 & 3 & 3.08 & 0.333 & 0.327 \\
780 & 3 & 3.25 & 0.333 & 0.338 \\
1060 & $\geq 6$ & 6.33 & $\leq 0.167$ & 0.155 \\
\hline
\end{tabular}

aThe fiber with $\lambda_{c}=1060 \mathrm{~nm}$ should theoretically propagate more than six modes.

bThe number of guided modes is given for the operating wavelength of $488 \mathrm{~nm}$ (Fig. 2).

Quantity $N_{\exp }$ is given by $\left.N_{\exp }=\langle\mathrm{J}\rangle / \mathrm{J}_{0}\right\rangle$, where $\mathrm{J}_{0}$ ) is the average signal measured for the fiber with $\lambda_{c}=488 \mathrm{~nm}$ (see text for details).

dTheoretical coherence factor $\mathrm{f}(\mathrm{N})$ is calculated by Eq. (19).

an EMI D191A photomultiplier) to be separated from the receiver unit. This is a major advantage to the classical pinhole receiver, which requires a bulky goniometer to provide satisfactory mechanical stability of the setup.

In addition, a long fiber provides an effective elimination of spurious higher-order modes. In our setup we used a fiber of $4 \mathrm{~m}$ length, which is sufficient to obtain satisfactory mode purity. The use of a fiberoptic receiver also provides a major advantage in the alignment of the spectrometer: a part of the excitation beam is coupled into the receiving fiber at its end pointing to the detector. The beam emerging from the receiving fiber is then intersected with the excitation beam in the center of the index-matching vat. This intersection defines the scattering volume. After connection of the receiving fiber to the photomultiplier, fine adjustment is performed when a maximal signal amplitude is looked for while the fiber is translated vertically across the probing beam profile in a strongly scattering sample.

Normalized intensity autocorrelation functions $\mathrm{g}^{2}(\tau)$ of a dilute suspension of highly monodisperse polystyrene latex particles of $91 \mathrm{~nm}$ nominal diameter have been measured with this setup by the use of fibers with different cutoff wavelengths $(\mathrm{OZ}$ fibers from Table 1). The resulting correlograms are shown in Fig. 6. From a second-order cumulant fit of field autocorrelation functions $g^{1 /}(\tau)$, an average hydrodynamic radius $R_{H}=40 \mathrm{~nm}$ was found, with a variation of $\sim 1 \%$. The numbers of guided modes, the coherence factors, and the average signals for the different fibers are shown in Table2. The experimental coherence factor of the single-and few-mode experiments is in good agreement with coherence factor $f(N)$ predicted by Eq. (19). This indicates that the effective radii $a_{0}$ of all fiber assemblies are sufficiently small to approximate the case of a laterally homogeneous source and that higher-order LP modes are uniformly transferred through the fiber. ${ }^{3}$
The usefulness of the spectrometer for static lightscattering experiments was investigated by the use of the same latex sample. From the angular dependence of the average intensity, the radius of gyration was determined by the use of the Guinier approximation. ${ }^{10} \mathrm{~F}$ or the polystyrene particles investigated, the relation $R_{G}=\sqrt{3 / 5} R_{H}$ was satisfied to within $7 \%$. This confirmed the consistency between the static and the dynamic light-scattering measurements. We did not go into further detail because the performance of single-mode fiber optics for static light scattering already has been convincingly demonstrated by Suparno et al. ${ }^{11}$

\section{Conclusions}

We have shown that the fiber receiver is indeed superior to the classical pinhole setup in terms of a high coherence factor and a high signal-collecting efficiency, thus corroborating the optimistic observations in Refs. 1, 4, and 11 . Our experimental data are in good quantitative agreement with the theory in Ref. 3. The use of fibers with different cutoff wavelengths allows us to optimize the average scattering signal and coherence factor by variation of the number of guided modes for a specific experimental situation. In addition, a simple experimental setup has been constructed and proved to be reliable and easy to handle under various experimental conditions.

This research was supported by the Swiss National Science Foundation. Wethank A. Hunziker for carefully reading the manuscript.

\section{References and Notes}

1. J . P. McClymer, "Comparison of multimode and single-mode optical fibers for quasi-elastic light scattering," Rev. Sci. Instrum. 61, 2001-2002 (1990).

2. B. Chu, Laser Light Scattering (Academic, New York, 1991).

3. J. Rička, "Dynamic light scattering with single-mode and multimode receivers," Appl. Opt. 32, 2860-2875 (1993).

4. R. G. Brown, "Dynamic light scattering using monomode optical fibers,"Appl. Opt. 26, 4846-4851 (1987).

5. E. G. Neumann, Single-ModeFibers (Springer, Berlin, 1988).

6. H. Wiese and D. Horn, "Single-mode fibers in fiber-optic quasielastic light scattering: a study of the dynamics of concentrated latex dispersions,"J . Chem. Phys. 94, 6429-6443 (1991).

7. H. S. Dhadwal and B. Chu, "A fiber-optic light scattering spectrometer," Rev. Sci. Instrum. 60, 845-853 (1989).

8. K. Schätzel, R. Kalström, B. Stampa, and J . Ahrens, "Correction of detection-system dead-time effects on photon-correlation functions,"J . Opt. Soc. Am. B 6, 937-947 (1989).

9. K. Schätzel, "Noise on photon correlation data," Quantum Opt. 2, 287-305 (1990).

10. The Guinier formula, $\langle\backslash Q / / J \backslash 0)=\exp \left[-Q^{2} R_{G}{ }^{2} / 3\right.$, is an approximation to the form factor of a homogeneous sphere, with a radius of gyration $\mathrm{R}_{\mathrm{G}}$ for $\mathrm{QR}_{\mathrm{G}}<1$.

11. K. Suparno, P. Deurloo, R. Stamatelopoulos, R. Srivastva, and J . C. Thomas, "Light scattering with single-mode fiber coll limators,"Appl. Opt. 33, 7200-7205 (1994). 